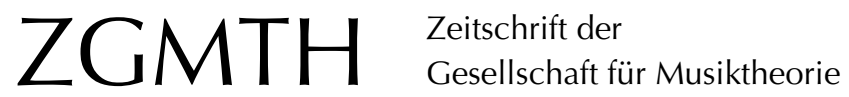

Kaiser, Ulrich (2014): Überlegungen zur Didaktik der Musiktheorie. ZGMTH 11/2, 191-208. https://doi.org/10.31751/742

(C) 2014 Ulrich Kaiser

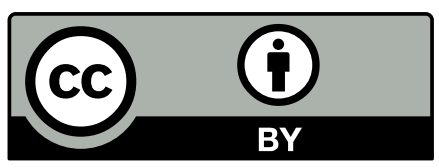

Dieser Text erscheint im Open Access und ist lizenziert unter einer Creative Commons Namensnennung 4.0 International Lizenz.

This is an open access article licensed under a

Creative Commons Attribution 4.0 International License.

veröffentlicht / first published: 24/07/2015

zuletzt geändert / last updated: 19/02/2016 


\title{
Überlegungen zur Didaktik der Musiktheorie
}

\author{
Ulrich Kaiser
}

\begin{abstract}
Nach einer methodisch orientierten Analyse von drei Lehrproben erfolgen Überlegungen zur Didaktik des Musiktheorieunterrichts sowie zum Methodikunterricht des Hauptfachs Musiktheorie. Diese Überlegungen nehmen Bezug auf aktuelle Themen des erziehungswissenschaftlichen Diskurses. Dazu gehören das viel diskutierte Thema ,Kompetenzorientierungı, die von Niklas Luhmann aufgezeigte Trivialisierungsproblematik und die derzeit wesentlich von John Hattie beeinflusste Diskussion zur Selbstevaluation Lehrender. Vor diesem Hintergrund wird ein kritisches Schlaglicht auf die gegenwärtige Praxis von Bewerbungsverfahren in der akademischen Musiktheorie geworfen.
\end{abstract}

A methodically oriented analysis of three demonstration lessons is followed by contemplations on didactics of music theory and the challenge of giving methodological instructions to music theory students. These contemplations refer to topics currently being discussed in the discourse on educational science, among them the much respected subject of 'Kompetenzorientierung', the problem of 'Trivialisierung' as demonstrated by Niklas Luhmann and the debate on selfevaluation of teachers, which is currently substantially influenced by John Hattie. Against this backdrop a critical light is cast on the current practice of application procedures in academic music theory.

Der folgende Beitrag besteht aus drei Teilen: Im ersten Teil werden drei Lehrproben beschrieben, die ich in Bewerbungsverfahren für Musikhochschulprofessuren im Fach Musiktheorie persönlich erlebt habe. Die ersten beiden Lehrproben sind dabei von allen Beteiligten sehr gut beurteilt worden und haben in einem Fall sogar zum Erhalt der ausgeschriebenen Stelle geführt. Die dritte Lehrprobe hingegen hinterließ weder bei der Kommission noch den Studierenden einen positiven Eindruck. Im zweiten Teil werden die beobachteten Unterrichtsarrangements dann vor dem Hintergrund aktuell diskutierter erziehungswissenschaftlicher Themen kritisch reflektiert und im Hinblick auf die jeweiligen Ziele analysiert. Die Ergebnisse dieser Analyse werden schließlich in einem dritten Teil auf die derzeitige Ausbildungssituation des Hochschulfachs Musiktheorie bezogen. Dadurch wird ein Dilemma sichtbar, das derzeit den Unterricht in Methodik/Didaktik des Hauptfachs Musiktheorie in einer sehr unglücklichen Weise prägt. 


\section{ERSTER TEIL}

\section{Erstes Beispiel: Unterrichtseinheit zum Thema ^Werkanalyse`}

Für diese Lehrprobe waren das Thema `Werkanalyse`sowie die Länge der Lehrveranstaltung (25 Min.) vorgegeben. Die Gruppe der zu unterrichtenden Studierenden war in ihrer Zusammensetzung (Größe, Vorkenntnisse sowie instrumentalpraktische Fähigkeiten der teilnehmenden Personen) der Lehrperson nicht bekannt. Der Bewerber hatte als Gegenstand der Werkanalyse das Vorspiel zum III. Akt der Oper La Traviata von Giuseppe Verdi gewählt. Die Lehrprobe lässt sich in vier Phasen beschreiben:

\section{Phase 1 - Informierender Unterrichtseinstieg}

Nach Informationen zum geplanten Unterrichtsverlauf wurde das Musikbeispiel angehört, verbunden mit dem Arbeitsauftrag, dabei in den Noten alles zu kennzeichnen, was für erwähnenswert erachtet wird.

\section{Phase 2 - Freies/diskursives Gespräch}

Es entwickelte sich eine recht lebhafte Diskussion, in der viele Einzelbeobachtungen zu verschiedenen Parametern der Komposition gesammelt werden konnten. ${ }^{2}$

\section{Phase 3 - Fragend-entwickelndes Gespräch ('Sokratischer Methode)}

Nach ungefähr zwei Dritteln der Lehrprobe wurde von der Lehrperson der von den Studierenden erwähnte chromatische Übergang von Takt 7 zu Takt 8 aufgegriffen und eine Frage nach der Weiterführung der Melodie gestellt. Der Zurückhaltung bei der Beantwortung dieser unpräzisen Frage folgte der Hinweis, dass die Chromatik ja am Taktübergang erklinge und man in einem ersten Schritt deshalb die nachfolgenden Taktübergänge untersuchen könne. Daraufhin wurde unter Berücksichtigung der Vorhalte der melodische Stufengang (c-)h-b-as-g-f-es (T. 7-11) als Struktur erkannt und - da in den Takten 12 und 14 das es jeweils auf der Takteins erklingt - die Harmonik Es-Dur/As-Dur als eine am Es saufgehängte، Pendelharmonik. Auf die Frage nach einer idealtypischen Weiterführung dieser Struktur wurde anstelle des erwarteten $d$ die chromatische Variante des entdeckt (T. 19-25) sowie die Tonfolge $c-h-c$ (Sopranklausel) als Struktur der abschließenden Kadenz (T. 28-31). Das Ergebnis dieser Phase war das folgende Modell zur Veranschaulichung des formalen Verlaufs der Einleitung zum III. Akt der Oper La Traviata (vgl. Beispiel 1):

1 »Ich rate Ihnen, möglichst häufig den so genannten sinformierenden Unterrichtseinstieg، zu praktizieren, also zu Beginn der Stunde kurz und ohne Schnörkel zu sagen bzw. an die Tafel zu schreiben, was auf die Schüler zukommt« (Meyer 2011, 36).

2 Thematisiert wurden im Hinblick auf die Instrumentation beispielsweise der einleitende hohe Streicherklang sowie die Weitung des Klangraums im Verlauf des Stücks (verbunden mit dem Hinzutreten der Holzbläser). Erwähnung fand auch die Änderung des Satzbilds beim Einsatz der tiefen Streicher (Bass mit nachschlagenden Streicherakkorden). Für den harmonischen Bereich wurden 


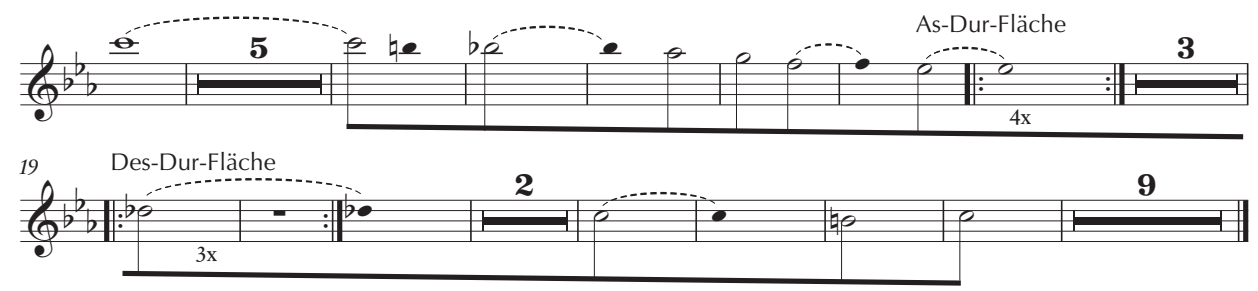

Beispiel 1: Giuseppe Verdi, La Traviata, III. Akt, Vorspiel (Andante), Tonleiterstruktur T. 1-31 (http://www.kaiser-ulrich.de/zgmth/kaiser-didaktikaufsatz-example-1.html)

\section{Phase 4 - Zusammenfassung und Ausblick}

Vom Bewerber wurde darauf hingewiesen, dass sich viele Kompositionen über eine Tonleiterstruktur ${ }^{3}$ erklären ließen und dass in diesem speziellen Fall zwei Strukturtöne mit längerer Ausdehnung auffielen. Die Ausarbeitung dieser harmonischen Bereiche - das am es saufgehängte`As-Dur sowie die neapolitanische Region Des-Dur - könnten dabei als für den weiteren Verlauf der Oper bedeutsam interpretiert werden. ${ }^{4}$ Den Abschluss bildete ein Ausblick auf mögliche Folgestunden. Die nachstehende Liste fasst den Ablauf der Lehrprobe und das Zeitmanagement zusammen:

1. Phase - Informierender Unterrichtseinstieg und Anhören des Analysebeispiels (5 Min.)

2. Phase - Freies/diskursives Gespräch zur gehörten Musik (10 Min.)

3. Phase - gelenktes Unterrichtsgespräch zur Tonleiterstruktur und Hinweis zu den Klangflächen As-Dur und Des-Dur (7 Min.)

4. Phase - Zusammenfassung und Ausblick (3 Min.)

Die Motivation und gute Beteiligung der Studierenden sowie das ansehnliche Arbeitsergebnis im Hinblick auf die zur Verfügung stehende Zeit führten, wie bereits erwähnt, zu einer insgesamt sehr guten Bewertung der Lehrprobe.

die funktionsharmonischen Zusammenhänge einiger Takte genannt, z.B. (D)-S-D-T in den Takten 8-11 und die große Schlusskadenz in den Takten 28-31. Melodisch fielen die Des-Dur-Kantilene (T. 19ff.), die zahlreichen Vorhalte sowie der besondere chromatische Übergang vom zweiten G-Dur-Halbschlussakkord (T. 7) in den kleinen C-Dur-Septakkord (T. 8) auf.

3 Locus classicus: J. S. Bach, Präludium C-Dur BWV 846. Im Bereich der Oper vgl. die Arie des Cavaradossi »E lucevan le stelle» aus Tosca von Giacomo Puccini, den Chor »Les voici! im letzten Akt (Nr. 26, Marsch und Chor, Takt 27ff. bzw. Ziffer 13) der Oper Carmen von George Bizet u.v. a.

4 Vgl. im III. Akt das Duett »Parigi, o cara« zwischen Violetta und Alfredo in As-Dur (T. 75 ff.) sowie im Finale den Abschnitt "Prendi, quest'è l'immagine" in des-Moll (T. 53ff.) und das sich dem "Se una pudica vergine« anschließende Quintett in Des-Dur (T. 83 ff.). 


\section{Zweites Beispiel:}

\section{Unterrichtseinheit zum Thema `Einführung in die romantische Harmonikı}

Die Vorgaben der folgenden Unterrichtseinheit waren das Thema sowie die Länge der Lehrveranstaltung (30 Min.). Dem Bewerber wurde auf der Einladung zur Lehrprobe schriftlich mitgeteilt, dass die zu unterrichtende Gruppe aus Studierenden der Lehramtsstudiengänge bestehen werde. Nicht bekannt waren dem Bewerber die Gruppengröße und in welchem Studiensemester sich die Studierenden befanden. Es wurden ein CDPlayer sowie 12 Harmonie-Karten (Din A6) eingesetzt, von denen hier zwei exemplarisch vorgestellt werden (vgl. Beispiel 2):
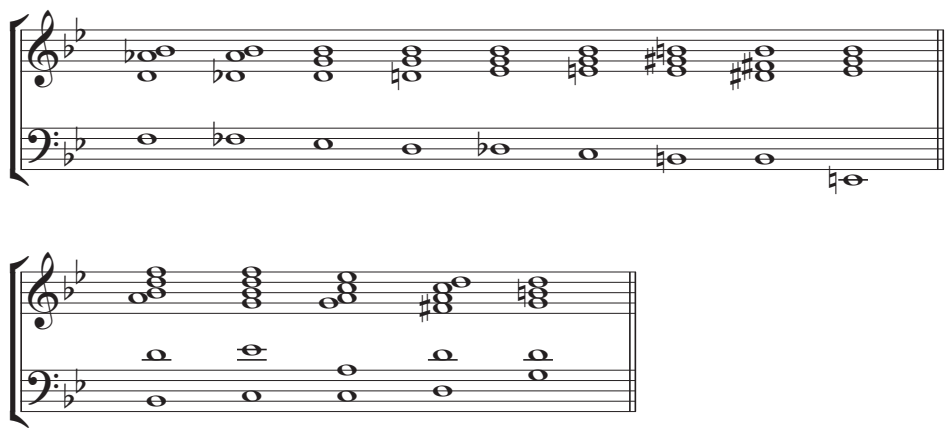

Beispiel 2: Zwei Harmoniekarten (Karten 1 und 4) zur Unterrichtseinheit 'Einführung in die romantische Harmonikı (http://www.kaiser-ulrich.de/ zgmth/kaiser-didaktikaufsatz-example-2.html)

Die Unterrichtseinheit lässt sich in fünf Phasen beschreiben:

Phase 1 - Informierender Unterrichtseinstieg

Nach einem Ausblick auf den Verlauf der Stunde wurden die Studierenden angehalten, in vier Gruppen jeweils drei Harmoniekarten zu analysieren und die jeweiligen Beispiele als »vorbarocke Harmonik«, »barocke Harmonik«, »klassisch-frühromantische Harmonik«, "spätromantische Harmonik« oder »nachromantische Harmonik« zu klassifizieren. Jede Harmoniefolge wurde vom Bewerber einmal am Klavier vorgespielt. Argumente für die jeweilige Klassifizierung einer Harmoniefolge sollten von den Studierenden schlagwortartig festgehalten werden.

\section{Phase 2 - Durchführung der Gruppenarbeit}

Es entwickelte sich unter den Studierenden eine lebhafte Diskussion. Die Lehrperson konnte zu jeder Zeit um Hilfe gebeten werden, wurde im Verlauf der Gruppenarbeit jedoch nur zweimal angesprochen. In dieser Phase der Lehrprobe ließen sich daher in erster Linie Aktivitäten der Studierenden beobachten, der Bewerber hielt sich die ganze Zeit im Hintergrund. 


\section{Phase 3 - Präsentation}

Von einem Gruppensprecher wurde das jeweilige Gruppenarbeitsergebnis vor dem Plenum erläutert, und die Mitglieder jeder Gruppe waren aufgefordert, eventuelle aus dem Plenum an sie gestellte Fragen zu beantworten. Dadurch bestand die Möglichkeit, durch kritische Einwände und Verteidigung ein Gruppenarbeitsergebnis zu bestätigen oder zu modifizieren. Die endgültige Klassifikation einer Harmoniefolge wurde abschließend in eine vom Bewerber an der Tafel vorbereitete Tabelle eingetragen. Das nachstehende Diagramm (Tabelle 1) zeigt die Gruppenarbeitsergebnisse für die beiden erwähnten Harmoniekarten:

\begin{tabular}{|l|l|l|l|l|l|}
\hline $\begin{array}{l}\text { Karten- } \\
\text { nummer }\end{array}$ & $\begin{array}{l}\text { vorbarocke } \\
\text { Harmonik }\end{array}$ & $\begin{array}{l}\text { barocke Har- } \\
\text { monik }\end{array}$ & $\begin{array}{l}\text { klassisch-früh- } \\
\text { romantische } \\
\text { Harmonik }\end{array}$ & $\begin{array}{l}\text { spät- } \\
\text { romantische } \\
\text { Harmonik }\end{array}$ & $\begin{array}{l}\text { nach- } \\
\text { romantische } \\
\text { Harmonik }\end{array}$ \\
\hline Karte 1 & & & & & $x$ \\
\hline Karte 4 & & & & $x$ & \\
\hline
\end{tabular}

Tabelle 1: Lehrprobe `Einführung in die romantische Harmonikı, Einschätzung der auf den Harmoniekarten abgebildeten Beispiele durch die Studierenden

\section{Phase 4 - Evaluation}

Die zu den Harmoniefolgen gehörigen Musikbeispiele wurden angehört und die Studierenden aufgefordert zu beurteilen, ob sie ihre Kategorisierung nach dem Anhören des jeweiligen Musikbeispiels beibehalten wollten oder nicht. Im Falle einer Änderung der Auffassung sollte diese begründet werden. Die erste Karte chiffriert den harmonischen Verlauf einer Stelle aus dem sechsstimmigen Madrigal Out from the Vale von John Ward (1571-1638):

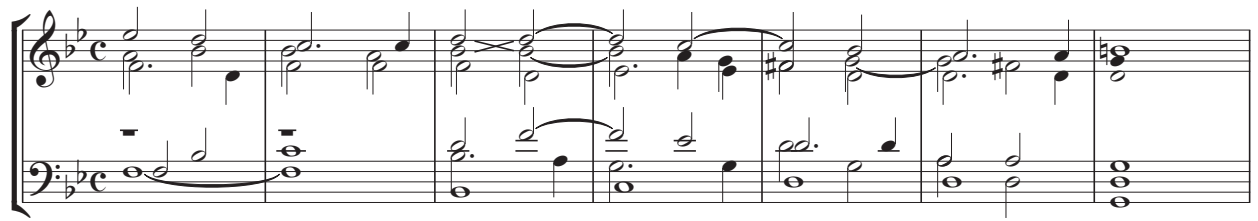

Beispiel 3: John Ward, Out from the Vale, T. 58-64

(http://www.kaiser-ulrich.de/zgmth/kaiser-didaktikaufsatz-example-4.html)

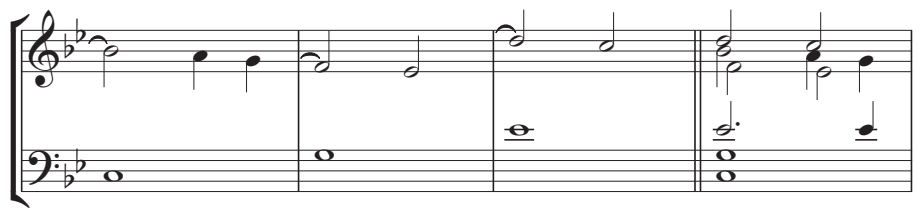

Beispiel 4: John Ward, Out from the Vale, kontrapunktische Erklärung der manieristischen Terzschichtung c-es-g- $b-d-f$ 
Der Undezimklang, der für die Studierenden den Anlass gab, das Beispiel im 20. Jahrhundert zu verorten, lässt sich in Kenntnis des Kontextes als manieristische Schichtung von drei Septimensynkopen verstehen, wobei jeder Ton des Dreiklangs c-es-g Bezugston (Agens) einer Septimensynkope (Patiens) ist (Beispiel 4).

Die fehlerhafte Kategorisierung dieses Beispiels wurde von den Studierenden erkannt und eigenständig korrigiert. Die zweite Karte steht für die virtuose Ausarbeitung eines Zirkelmodells in einer Klavierkomposition von Franz Liszt:
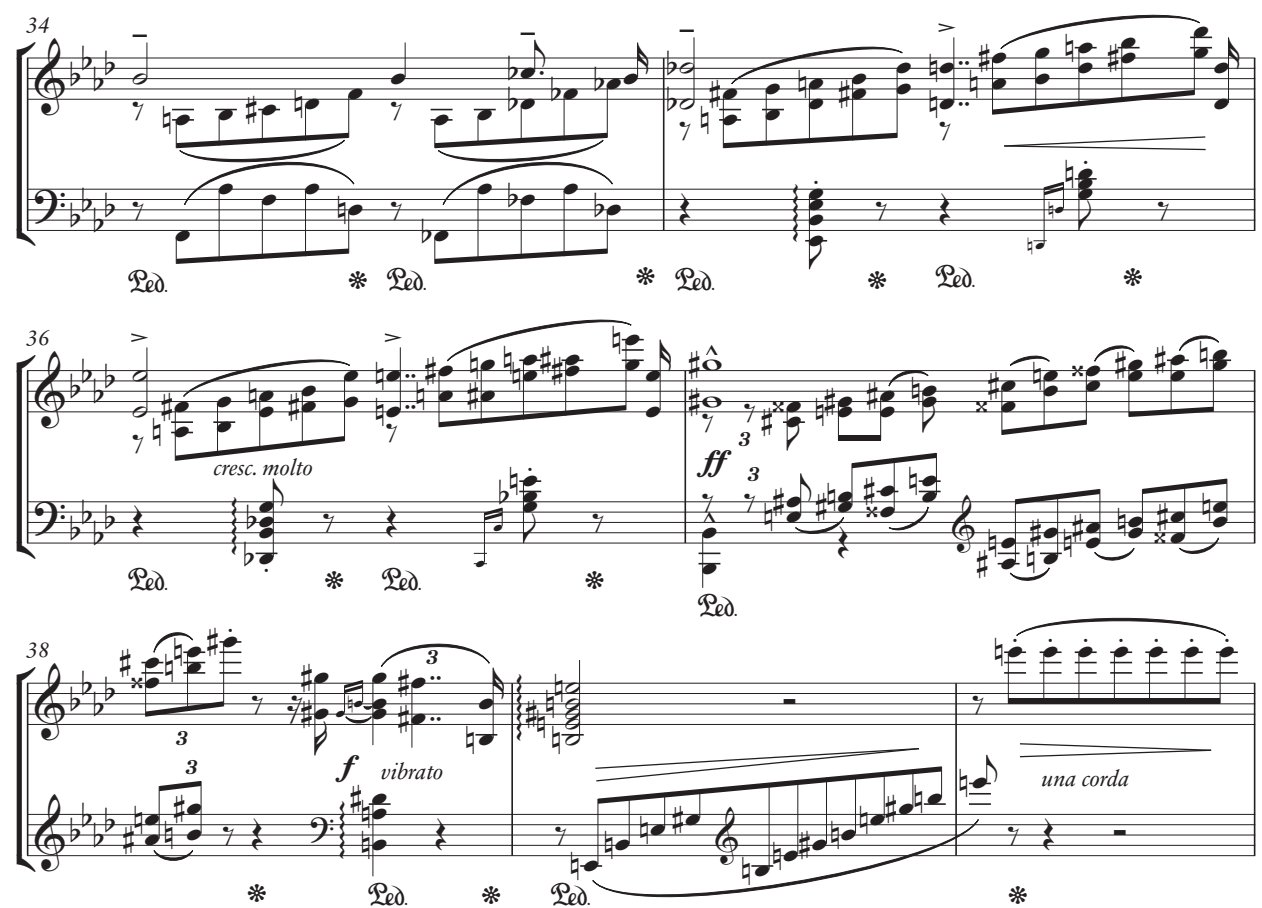

Beispiel 5: Franz Liszt, Années de Pèlerinage. Deuxième Année: Italie, Sonetto del Petrarca Nr. 123, T. 34-40 (http://www.kaiser-ulrich.de/zgmth/kaiser-didaktikaufsatz-example-6.html)

In diesem Fall bestätigten die Studierenden die von ihnen vorgenommene Kategorisierung.

\section{Phase 5 - Feedback}

Das Feedback der Lehrperson auf die Gruppenarbeitsergebnisse bestand in der Frage, warum einige Klassifizierungen der zwölf Karten zuerst fehlerhaft vorgenommen worden waren und nach Anhören der originalen Kompositionen relativ einfach korrigiert werden konnten. Die Studierenden antworteten, dass der isolierte Parameter Harmonik ohne Kontext und gattungsspezifische Informationen nicht aussagekräftig sei. Darüber hinaus wurde die Vorstellung als Klischee erkannt, dass alte Musik grundsätzlich einfacher als romantische Musik sei und dass die Vorstellung einer progressiven Entwicklung von »ba- 
rocker« zu »klassischer« und »romantischer« Harmonik ein unzureichendes Musik- und Geschichtsbild transportiere. Die folgende Übersicht fasst den Stundenverlauf und das Zeitmanagement zusammen:

1. Phase - Vorbereitung (4 Min.)

2. Phase - Durchführung der Gruppenarbeit (10 Min.)

3. Phase - Präsentation und Plenumsgespräch (5 Min.)

4. Phase - Evaluation durch Anhören der Musikbeispiele und kurzes Statement der Studierenden (6 Min.)

5. Phase - Feedback und Kommentierung (5 Min.)

\section{Drittes Beispiel:}

\section{Evaluation einer Vorgehensweise zum Schreiben eines Choralsatzes}

Dem Bewerber waren als Thema eine ssatztechnische Lehrprobe mit fortgeschrittenen Studierenden sowie die Länge der Lehrveranstaltung (30 Min.) vorgegeben. Als Gegenstand der Unterrichtsdemonstration wurde vom Bewerber die Ausarbeitung eines Choralsatzes bzw. die Bearbeitung der ersten beiden Choralzeilen des Chorals O Ewigkeit, Du Donnerwort gewählt. Die Lehrprobe verlief in fünf Phasen.

Phase 1 - Informierender Unterrichtseinstieg

Den Studierenden wurde mitgeteilt, dass es verschiedene bereits publizierte Vorgehensweisen gebe und sie in dieser Unterrichtseinheit in Einzelarbeit testen sollten, ob sich über eine vom Bewerber mitgebrachte neue Methode »in drei Schritten« das Schreiben eines Choralsatzes erlernen lasse oder nicht. Nach dem Testen und Vorliegen von Arbeitsergebnissen sollte die Vorgehensweise diskutiert und sowohl in ihren Stärken als auch Schwächen reflektiert werden. Die Regeln für die drei Arbeitsschritte sowie Arbeitsbögen für jeden einzelnen Arbeitsschritt wurden den Studierenden vom Bewerber zur Verfügung gestellt.

\section{Phase 2 - Erster Arbeitsschritt}

Hier ging es darum, mithilfe einer Tabelle jedem Melodieton der vorgegebenen Choralzeilen eine Harmonie zuzuweisen. Dabei waren nach Möglichkeit a) Harmoniewiederholungen und b) die Subdominante nach einer Dominante zu vermeiden.

\begin{tabular}{|l|l|l|l|l|l|l|l|l|}
\hline Tonleiterstufe & 1 & 2 & 3 & 4 & 5 & 6 & 7 & 8 \\
\hline Funktion & $\mathrm{T}$ & $\mathrm{D}$ & $\mathrm{T}$ & $\mathrm{S} / \mathrm{D} \downarrow$ & $\mathrm{T} / \mathrm{D}$ & $\mathrm{S}$ & $\mathrm{D}$ & $\mathrm{T}$ \\
\hline
\end{tabular}

Tabelle 2: Lehrprobe `Choralsatz‘, Harmonisierung von Melodiestufen innerhalb einer Tonart

Nach kurzer Stillarbeit wurde das einheitliche Ergebnis an der Tafel fixiert: 


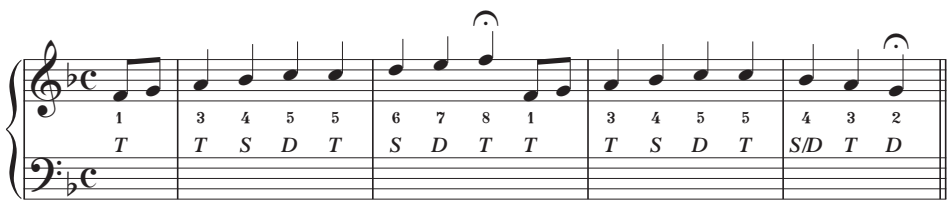

Beispiel 6: Lehrprobe `Choralsatz‘, Ergebnis Schritt 1: Harmonisierung der Töne der Choralmelodie

\section{Phase 3 - Zweiter Arbeitsschritt}

Im zweiten Arbeitsschritt sollte zu dieser Harmonisierung ein Bass unter Berücksichtigung der folgenden Regeln ausgearbeitet werden:

- Verwenden Sie im Zeileninnern Terzen, Sexten und die verminderte Quinte

- und an den Zeilenenden Quinte oder Oktave.

- Ergänzen Sie anschließend bei allen Terzsprüngen im Bass einen Durchgang.

In Verbindung mit der Harmonievorgabe hätte dieser Arbeitsauftrag zu dem folgenden Ergebnis führen können:

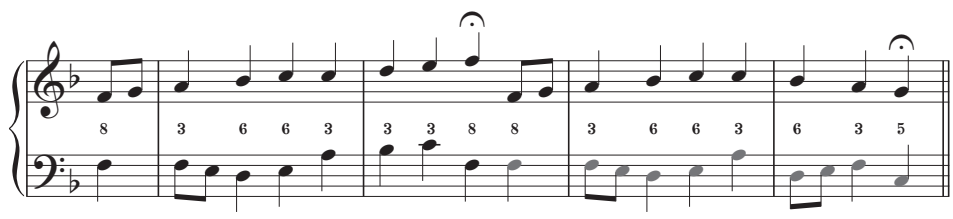

Beispiel 7: Lehrprobe `Choralsatz‘, Ergebnis Schritt 2: Erstellen einer Bassstimme

Dieses Arbeitsergebnis wurde jedoch nicht erreicht, lediglich die erste Choralzeile konnte mit einem Bass versehen werden. Der Zeitverlust resultierte aus einer Bemerkung der Lehrperson gegenüber einer Studentin, ihr Bass sei nicht mit der Regelvorgabe vereinbar. Aus diesem Grunde werde es ihr später nicht möglich sein, die Vorgehensweise zu evaluieren. Diese Bemerkung löste bei der Studentin spürbaren Unwillen aus, da ihr anscheinend am Schreiben eines Choralsatzes nach eigener Vorstellung gelegen war, während es dem Bewerber auf die Evaluierung der Vorgehensweise ankam. Den Zeitverlust versuchte der Bewerber durch eine Beschränkung auf die erste Choralzeile, sowie den Wechsel von Einzelarbeit zu Gruppenarbeit aufzufangen.

\section{Phase 4 - Dritter Arbeitsschritt}

Ergänzen Sie die Mittelstimmen Alt und Tenor. Vermeiden Sie dabei:

- offene Parallelen (Oktave, Quinte, Einklang),

- Terzverdoppelung in dominantischen Klängen, 
- unnötige Bewegungen (im Alt oder Tenor),

- unvollständige Akkorde (ohne Quinte oder Terz) und

- Quartsextakkorde.

Beim Ergänzen der Mittelstimmen ergaben sich relativ schnell die in Beispiel 8 grau markierten Parallelen in perfekten Konsonanzen.

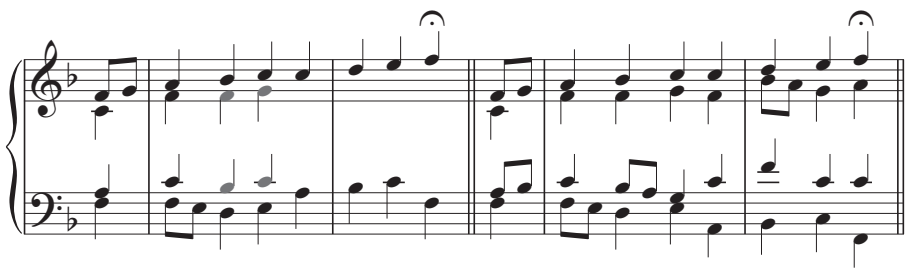

Beispiel 8: Lehrprobe `Choralsatzı, Ergebnis Schritt 3: einfache Mittelstimmenaussetzung

An dieser Stelle der Lehrprobe wies der Bewerber nicht auf die Standardlösung für dieses bekannte Problem hin (Terzverdoppelung in der Subdominante), sondern ließ die Studierenden eine Lösung eigenständig erarbeiten (Beispiel 8 rechts). Die Ausarbeitung des in der Tenorführung ungeschickten Satzes, an dessen Klaviervorspiel der Bewerber dann noch zu guter Letzt scheiterte, benötigte relativ viel Zeit.

\section{Phase 5 - Kritik und Reflexion}

Durch den Zeitverlust blieb kein Raum zur Evaluation der Vorgehensweise. Der Bewerber unterbrach deshalb wenige Minuten vor Schluss die Lehrprobensituation und erläuterte den Studierenden sowie der Kommission, dass die Evaluation der Vorgehensweise eigentlich Kern der Lehrdemonstration hätte sein sollen, diese aber aufgrund des Standes der Choralsatzausarbeitung sowie der fortgeschrittenen Zeit nicht mehr in der Lehrprobe sinnvoll möglich sei. Die Lehrprobe endete für alle Seiten unbefriedigend.

\section{ZWEITER TEIL:}

\section{AKTUELLE THEMEN DER ERZIEHUNGSWISSENSCHAFTEN}

Schulleistungsuntersuchungen wie PISA ${ }^{5}$, TIMSS $^{6}$ und IGLU ${ }^{7}$ sowie Publikationen aus der Erziehungswissenschaft und Soziologie haben in den letzten Jahren die Musikpädagogik

5 PISA sind internationale Schulleistungsuntersuchungen der OECD (das Kürzel wird in verschiedenen Sprachen unterschiedlich aufgelöst). Seit 2000 werden diese Untersuchungen regelmäßig (alle drei Jahre) durchgeführt, PISA-Ergebnisse erhielten in der Vergangenheit zum Teil hohe mediale Aufmerksamkeit.

6 TIMSS steht für sTrends in International Mathematics and Science Study und bezeichnet eine vergleichende Schulleistungsuntersuchung auf internationaler Ebene, die von der IEA (`International Association for the Evaluation of Educational Achievement`) regelmäßig (alle vier Jahre) durchgeführt wird. 
stark beeinflusst. ${ }^{8}$ Irritierend wirken sich seit einigen Jahren darüber hinaus die Veröffentlichungen des neuseeländischen Pädagogen und Erziehungswissenschaftlers John Hattie aus $^{9}$, während die Systemtheorie Niklas Luhmanns bzw. seine Beiträge zur Pädagogik ${ }^{10}$ im musikpädagogischen Diskurs nur vereinzelt aufgegriffen worden sind. ${ }^{11}$ Ebenfalls stark geprägt wird die derzeitige Diskussion vom Begriff der `Kompetenzorientierung ‘. Unter Kompetenz werden im vorliegenden Aufsatz und in Übereinstimmung mit dem Erziehungswissenschaftler und Psychologen Franz Weinert »die bei Individuen verfügbaren oder von ihnen erlernbaren kognitiven Fähigkeiten und Fertigkeiten« verstanden, »bestimmte Probleme zu lösen, sowie die damit verbundenen motivationalen, volitionalen und sozialen Bereitschaften und Fähigkeiten, die Problemlösungen in variablen Situationen erfolgreich und verantwortungsvoll nutzen zu können «. ${ }^{12}$ Der Kompetenzbegriff nimmt also den Menschen und seine Fähigkeit der Wissensanwendung in unvorbereiteten Situationen in den Blick, welche von vielen Faktoren abhängt und sich nicht zwangsläufig aus erlerntem Fachwissen ergibt. Auch der Begriff der >Lernzielorientierung`, der wie die Kompetenzorientierung den `Outcome` pädagogischer Bemühungen fokussiert, hat derzeit wieder Konjunktur. Allerdings scheint nicht immer ganz klar zu sein, worin genau sich die Begriffe Kompetenz- und Lernzielorientierung in ihrem heutigen Sprachgebrauch unterscheiden. ${ }^{13}$ Eine gegenüber Kompetenz- und Lernzielorientierung untergeordnete Rolle spielt in der aktuellen musikpädagogischen Debatte der Begriff des vom Lehrenden vorgesehenen `Unterrichtsziels«. In Bezug auf den Musikunterricht findet er sich, wenn überhaupt, nur in überwiegend praktisch ausgerichteten Handleitungen. Verwendet man die Begriffe Kompetenz und Unterrichtsziel als Bezugspunkte, um die im Vorangegangenen beschriebenen Lehrproben zu analysieren, fallen strukturelle Unterschiede auf:

7 IGLU ist Abkürzung für die ıInternationale Grundschul-Lese-Untersuchung` (international bezeichnet als PIRLS `Progress in International Reading Literacy Study`). Die IGLU gibt es seit 2001, Deutschland ist seit der ersten Erhebung an dieser Untersuchung beteiligt.

8 »Ich folge in diesem Buch diesem Sprachgebrauch und stelle fest: Ich muss auf meine alten Tage umlernen. Die Über- und Unterlegenheit bestimmter Unterrichtskonzepte lässt sich zurzeit empirisch nicht nachweisen.« (Meyer 2011, 8)

9 Insbesondere Visible Learning (2009) und Visible Learning for Teachers (2013). Beide Publikationen sind in überarbeiteter Form auf Deutsch erschienen (Hattie 2014 und 2015).

10 Luhmann/Schorr 1982 und 1988 sowie Luhmann 2002 und 2004.

11 Zu erwähnen sind hier insbesondere die Arbeiten von Verena Weidner (u.a. Weidner 2012 und Druck i.V. a). Einen Überblick über die musikpädagogische Rezeption der Luhmannschen Systemtheorie gibt sie in ihrem systematischen Aufriss zum Verhältnis der beiden Theorietraditionen (Druck i. V. b).

12 Bundesministerium für Bildung und Forschung 2007, 72.

13 »Einige Autoren behaupten, kompetenzorientierte Bildungsstandards seien etwas ganz anderes als Lernziele. Aber wenn man sie fragt, worin denn der Unterschied liege, kommen sie ins Stocken. Deshalb behaupte ich: Jeder Bildungsstandard kann durch rein formale Umformung in ein Lernziel verwandelt werden. Man muss nur den Satz >Die Schüler sollen ... können` hinzufügen« (Meyer 2007, 170). Es finden sich allerdings schon ernst zu nehmende Versuche begrifflicher Differenzierung; vgl. z. B. für die Religionspädagogik Bosold/Eisele/Jakobs/Rueß/Scholz/Schwefel o. J. [online]. 
1. Für die Lehreinheit zum Vorspiel des III. Akts der Oper La Traviata von Guiseppe Verdi war von der Lehrperson ein Unterrichtsziel geplant, das durch direkte Instruktion ${ }^{14}$ erreicht wurde.

2. Die Lehrprobe zur Einführung in die romantische Harmonik war ebenfalls auf ein Unterrichtsziel hin ausgerichtet, das jedoch durch Gruppenarbeit ${ }^{15}$ angesteuert wurde.

3. Die dritte Lehrprobe hatte kein Unterrichtsziel im Sinne einer bestimmten Choralsatzausarbeitung, sondern diese sollte Grundlage einer Evaluation des methodischen Vorgehens und Handelns der Studierenden sein.

Zu 1.) Das von der Lehrperson geplante Unterrichtsziel der ersten Lehrprobe war die Einsicht der Studierenden in die musikalische Struktur und Form des Vorspiels zum III. Akt der Oper La Traviata. Dieses Ziel wurde im Zeitplan erreicht und die Studierenden hatten sich aktiv beteiligt. Fraglich ist hingegen, ob durch diesen Unterricht ein Kompetenzgewinn erzielt wurde, denn es dürfte Studierenden kaum möglich sein, selbstständig, also ohne Unterstützung durch eine Lehrperson, zu erkennen, dass sich der Verlauf von mehr als 30 Takten über eine Tonleiterstruktur beschreiben lässt. ${ }^{16}$ Die positive Beurteilung dieser Lehrprobe lässt sich also nicht auf einen Kompetenzzuwachs gründen, sondern dürfte auf der Anerkennung der Stimmigkeit von Inhalt und Methode sowie des gelungenen Zeitmanagements beruhen. Immerhin ist das Arbeitsergebnis an der Tafel für $25 \mathrm{Mi}$ nuten Lehrdemonstration erstaunlich, wenn man bedenkt, dass sich Unterrichtsverläufe nur bedingt vorhersehen lassen. Denn psychische Systeme (im Luhmann'schen Sinne), auch die von Studierenden, liefern selbst bei methodisch hervorragend geplantem Input keinen mit Sicherheit vorhersehbaren Output (oder in der Sprache Luhmanns: psychische Systeme sind keine `Trivialmaschinen`). Der Gesellschaftstheoretiker merkt hierzu pointiert an:

In den Ohren der Pädagogen mag es schrecklich klingen, wenn man ihr Geschäft als Trivialisierung der Menschen beschreibt. [...] Der Lehrer bzw. Prüfer stellt eine Frage, obwohl er die Antwort schon weiß. Das ist im sozialen Alltag unüblich und, wenn es herauskommt, peinlich. In der Schule [und man möchte ergänzen: auch in der Hochschule, Anm. UK] ist dies ein Standardverfahren der Kontrolle der Trivialisierung. ${ }^{17}$

14 »Als Direkte Instruktion (direct instruction) wird ein eher lehrerzentrierter, überwiegend frontal organisierter Unterricht bezeichnet.« (Meyer 2011, 8; Hervorhebung original)

15 »So gilt es als erstes zu beachten, dass Gruppenarbeit in 4 Phasen zu unterteilen ist: 1. Vorbereitungsphase[,] 2. Durchführung[,] 3. Präsentation/Auswertung[,] 4. Feedback/Evaluation[.] Hält man sich an diese Reihenfolge und führt die Gruppenarbeit professionell gesteuert durch, dann ist ein erfolgreicher Abschluss sowohl für die Schülerlnnen als auch für die Lehrerlnnen sehr wahrscheinlich.» (Reich 2014 [online], http://methodenpool.uni-koeln.de/download/gruppenarbeit.pdf, 1)

16 Auch, dass sich durch Betrachtung mehrerer Stücke mit vergleichbarer Struktur aus verschiedenen Epochen eine Strukturgeschichte konstruieren lässt, ist für Studierende der Lehramtsstudiengänge nicht gerade eine naheliegende Erkenntnis.

17 Luhmann 2002, 77f. (Hervorhebung original). 
Die Anregungen von Luhmann wurden in der Pädagogik beispielsweise von Annette Scheunpflug aufgegriffen, die den gleichen Sachverhalt über das Paradox zwischen individueller Freiheit und Unterrichtskausalität erläutert:

Der Bildungsbegriff markiert die Selbsttätigkeit des Individuums im Lernvorgang und damit die Unverfügbarkeit des Lernens aus der Perspektive des Lehrenden, während hingegen Unterrichtsplanungsmodelle als Theorie des Lehrens Unterrichtskausalität voraussetzen und eine Nichtbeliebigkeit des Unterrichtsarrangements suggerieren. ${ }^{18}$

Zwar gibt es Faktoren wie Organisation ${ }^{19}$ und Rollenasymmetrie ${ }^{20}$, die bestimmte Antworten im Unterricht wahrscheinlicher machen als andere, aber die Möglichkeit kontingenter Antworten bleibt als Problem der Unterrichtsplanung bestehen.

Für den lehrerzentrierten und frontal organisierten Unterricht hat Clemens Kühn die Bedeutung einer guten Fragetechnik betont. ${ }^{21}$ Mit sguter Fragetechnikı ist natürlich nicht eine schlechte Form der »Osterhasendidaktik ${ }^{22}$ gemeint, die Lernende so konditioniert wie einst der pensionierte Gymnasiallehrer von Osten sein Pferd, aber es dürfte fraglich sein, ob sich eine ungewollte Beeinflussung der Befragten überhaupt verhindern lässt. ${ }^{23}$ Gemeint ist vielmehr eine Fragetechnik, mit der die Kontingenz möglicher Antworten einschränkt wird, indem Fragen zu Antworten, die für den Fortgang der Stunde unentbehrlich sind, so präzise gestellt werden, dass es nur eine srichtige` Antwort gibt (Abbildung 1).

Aus dieser Perspektive waren in der Traviata-Lehrprobe zwei Fragen für das Erreichen des Unterrichtsziels entscheidend: Die Frage nach den Tonhöhen bei den Taktübergängen (T. 7-8 des Vorspiels zum III. Akt) und die nach den fehlenden Skalentönen zur Vervollständigung der Tonleiterstruktur. Beide Fragen ließen jeweils nur eine richtige

18 Scheunpflug 2004, 69.

19 »Wenn ein Lehrer morgens pünktlich zum Unterricht erscheint, richtet er sich, wie seine Kollegen auch, nach dem Stundenplan. Der Stundenplan ist das Resultat (der Kommunikation) einer Entscheidung. Insofern braucht der Lehrer nicht auf den Fluren umherzuirren und zu versuchen, irgendwo mit seinen Ideen akzeptiert zu werden; und nur so kann er seinen Unterricht vorbereiten bzw. sicher sein, daß ein Improvisieren genügt. Die gute Absicht zu erziehen, gewinnt mit Hilfe von Organisation Form« (Luhmann 2002, 160).

20 »Das wichtigste dürfte die Rollenasymmetrie sein, die sich in einer als Erziehung verstandenen Interaktion nicht umkehren läßt. Es muß geklärt sein, wem die Absicht [`Absicht zu erziehen`, Anm. UK] zugeschrieben wird und wem nicht. Damit ist zugleich das Problem der doppelten Kontingenz gelöst. Der Erzieher mag damit rechnen, daß der Zögling sich seiner Einwirkung zu entziehen sucht, nicht aber damit, daß der Zögling mit Gegenerziehung reagiert. Erziehung verträgt, anders gesagt, keine Rückkehr zur offenen Situation der doppelten Kontingenz. Sie ist, um dies auszuschließen, auf ausreichende Vorverständigungen und das heißt nicht zuletzt: auf gesellschaftliche Institutionalisierung angewiesen.« (Luhmann 2002, 55)

21 »Die philosophisch-pädagogisch-menschliche Kunst von Sokrates bestand in der Haltung, nicht vorzugeben, etwas zu wissen, jedoch andere in forschend fragenden Dialogen zu Einsichten zu führen. Er verstand sich als Geburtshelfer, nicht als Prediger. Mit 'Sokrates-Prinzip meine ich dies als (für mich persönlich allerwichtigste) pädagogische Idee: Schüler selbst Dinge entdecken zu lassen." (Kühn 2006, 44; Hervorhebung original)

22 Reich 2005, 182.

23 Vgl. hierzu Kaiser 2010, $199 f$. 

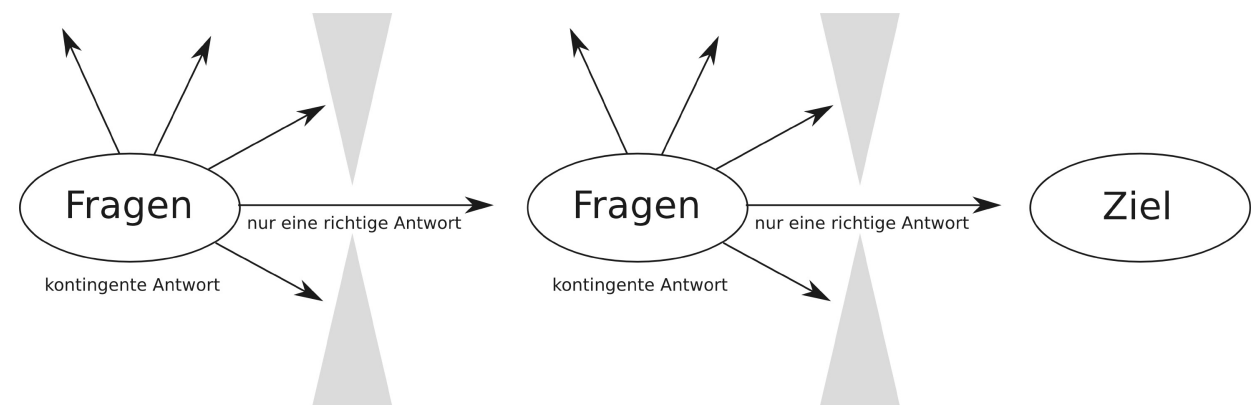

Abbildung 1: Erreichen eines Unterrichtsziels über trivialisierende Fragestellungen

Antwort zu und wurden in der Planung zu bestimmten Zeitpunkten platziert. Das musikanalytische Gespräch vor diesem Zeitpunkt war selbstverständlich sinnvoll, im Hinblick auf das Unterrichtsziel jedoch kaum von Bedeutung. Verallgemeinernd lässt sich sagen, dass das Erreichen eines Unterrichtziels im Rahmen eines lehrerzentrierten und frontal organisierten Unterrichts dann wahrscheinlich ist, wenn es sich über eine Kette trivialisierender Fragen herbeiführen lässt. Trivialisierende Fragen müssen dabei nicht notwendig einfach, sondern können sehr komplex sein ${ }^{24}$, denn auch komplexe Fragen sind trivialisierend, sofern sie lediglich eine korrekte Beantwortung zulassen. Diese Perspektive offenbart aber auch, dass die Phasen des freien/diskursiven und des fragendentwickelnden Gesprächs in der Lehrprobe nicht auseinander hervorgingen. Überspitzt gesagt war es egal, welche analytischen Beobachtungen von den Studierenden während der freien Gesprächsphase artikuliert wurden; in jedem Fall hätte der Bewerber zum angemessenen Zeitpunkt die Frage nach den Taktübergängen gestellt und damit die Aufmerksamkeit auf das Unterrichtsziel gelenkt. Jenseits einer Lehrprobensituation könnte ein solches Nichtaufgreifen studentischer Beiträge sogar als unbewusster Ausdruck einer Geringschätzung interpretiert werden.

Zu 2.) Das zur ersten Lehrprobe Gesagte trifft - obwohl sich am Ende des Unterrichts das gewünschte Aha-Erlebnis einstellte - in weiten Teilen auch auf die zweite Lehrprobe zu. Ziel der Gruppenarbeit war das Reflektieren des Parameters Harmonik anhand abstrakter Harmoniefolgen, das Ziel des Unterrichts die Einsicht in die Differenz zwischen abstrakter Harmoniefolge und konkretem kompositorischen Kontext. Unterrichtsziel und Gruppenarbeitsergebnisse waren also nur lose gekoppelt, nahezu alle denkbaren Gruppenarbeitsergebnisse hätten in das Unterrichtsgeschehen integriert werden können. Lediglich eine Ablehnung des Arbeitsauftrages (zum Beispiel die Bemerkung, dass sich aufgrund abstrakter Harmoniefolgen eine historisch-stilistische Einordnung nicht sinnvoll vornehmen lässt) oder durchgängig zutreffende stilistische Einordnungen (was ein sehr

24 »Trivialmaschinen sind solche, die auf einen bestimmten Input mit Hilfe einer eingebauten Funktion (der `Maschine $\triangleleft$ ) einen bestimmten Output produzieren. Ein anderer Input würde, sofern im Resonanzbereich der Maschine liegend, zu einem anderen Output führen. 2 mal 2 ist ........ 4; 2 mal 3 ist ....... 6. Die Maschine kann durch geeignete Programmierungen zu hoher Komplexität ihrer möglichen Inputs und Outputs gebracht werden. Das ändert nichts an ihrer Trivialität.« (Luhmann 2002, 77) 
unwahrscheinlicher Zufall gewesen wäre) hätten das Erreichen des Unterrichtsziels oder die Einhaltung der Zeitvorgaben gefährden können. Die insgesamt positive Bewertung der Lehrprobe dürfte hier ebenfalls auf Anerkennung der Methodenwahl, die eine gute Beteiligung der Studierenden bewirkte, sowie des gelungenen Zeitmanagements beruhen.

$\mathrm{Zu}$ 3.) Die dritte Lehrprobe hatte demgegenüber eine andere Anlage und aus Sicht der Kompetenzorientierung ein größeres Potenzial als die anderen beiden Lehrdemonstrationen. Denn die Studierenden sollten sich selbst in Ihrer Arbeitsweise beobachten und Arbeitsergebnisse einer dogmatischen Vorgehensweise evaluieren. Da die Regeln in verschiedenen Tonarten angewendet werden können, bieten sie wesentlich mehr Möglichkeiten, als auf den ersten Blick ersichtlich sind. ${ }^{25}$ Die Studierenden konnten ihre Vorkenntnisse hinsichtlich des Ausarbeitens von Satzaufgaben sowie der Oktavregel einbringen. Im Evaluationsgespräch hätten dabei vorhandene Fähigkeiten artikuliert, nicht erkannte Möglichkeiten der Methode entdeckt sowie Phänomene, die sich über die Regeln nicht realisieren lassen, benannt werden können (z. B. Standardkadenzen oder Sequenzen). In jedem Fall hätte ein solches Gespräch einen reflektierten Umgang mit vorhandenem und neu erworbenem Wissen eingefordert, der eine mechanische Anwendung oder Übernahme dieses Wissens ausgeschlossen hätte.

Das Misslingen der Lehrprobe vollzog sich auf verschiedenen Ebenen. Zum einen lag ein Fehler in der Planung, zwei Lehrproben in einer stattfinden zu lassen: eine Lehrprobe zur regelgeleiteten Herstellung eines Choralsatzes und eine weitere zur Evaluierung dieser regelgeleiteten Vorgehensweise. Vielleicht wäre es aus inhaltlicher Sicht geboten gewesen, auch die Ausarbeitungen (zusätzlich zu den Regeln) vorzugeben, um gleich in das Evaluationsgespräch einsteigen zu können. Formal hätte das allerdings eine flexible Auslegung der Vorgabe ssatztechnische Lehrprobe` erfordert. Zum anderen ließen sich die Studierenden nur bedingt darauf ein, eine unkritische Anwendung der Regeln zu versuchen und dabei eine kritische Beobachterrolle ihres Handelns einzunehmen. Das wiederum könnte sowohl an einem unklaren Unterrichtseinstieg des Bewerbers gelegen haben als auch an einer Überforderung der Studierenden in Anbetracht einer ungewohnten Aufgabenstellung. Nicht zuletzt war der Bewerber auf die Situation nicht vorbereitet, dass sfortgeschrittene Studierender an einer einfachen Mittelstimmenaussetzung bzw. bekannten Satzfehler-Konstellation scheitern.

\section{DRITTER TEIL:}

\section{ZUR SITUATION DES HOCHSCHULFACHS MUSIKTHEORIE}

Theorie sagt und Praxis zeigt, dass der Verlauf von Unterricht nicht vorhersehbar ist. Aus diesem Grunde ist gelungener Unterricht ein Glücksfall. Welche Konsequenzen resultieren daraus für das Hochschulfach Musiktheorie und seine Didaktik? Natürlich könnte man die Auffassung vertreten, dass, wenn Unterricht nicht vorhersehbar sei, er auch

25 Da eine ausführliche Erörterung der Möglichkeiten der Vorgehensweise, die auf einer didaktischen Modifikation der >Regola dell'ottava ‘ beruht, an dieser Stelle zu weit führen würde, sei auf ein online verfügbares eLearning-Tutorial verwiesen: http://www.musikanalyse.net/tutorials/choralsatz (Kaiser o. J. [online]). 
nicht geplant werden müsse. Wie unsinnig diese Auffassung ist, zeigt ein Vergleich: Auch das gelungene Konzert des Künstlers ist ein Glücksfall. Ein unerwartetes Geräusch, eine schwierige Raumakustik, eine kleine Unkonzentriertheit oder einfach nur ein schlechter Tag: Alles lässt sich im Nachhinein als Motiv des Misslingens bestimmen. Aber niemand käme wohl auf die Idee, den Wert des Übens oder einer Konzertvorbereitung anlässlich eines missglückten Auftritts in Frage zu stellen. Und es lassen sich noch weitere Parallelen zwischen Unterrichts- und Konzertsituation herstellen: Zum Beispiel mag der Applaus des Publikums schmeicheln, aber mindestens ebenso wichtig dürften für die Bewertung der Konzertleistung die nachträgliche Analyse der Künstlerin bzw. des Künstlers selbst und die öffentliche Kritik sein. Der Selbstanalyse und der öffentlichen Kritik entsprechen in der Didaktik Reflexion und Evaluation. Was Schülerinnen, Schüler und Studierende tatsächlich lernen, lässt sich nicht am Unterricht ablesen, sondern nur über professionelle Evaluationen ermitteln. Deswegen sind für John Hattie auch nicht Lehrpersönlichkeit, Unterrichtskonzepte oder Unterrichtserfahrungen entscheidend, sondern insbesondere die Bereitschaft der Lehrpersonen, die Wirkung ihres Unterrichtens auf das Lernen zu evaluieren:

Was ich nicht sage, ist, dass es »auf die Lehrperson ankommt«: Dieses Klischee wird von den in Lernen sichtbar machen zusammengetragenen empirischen Belegen in keiner Weise gestützt. Es ist ein Klischee, das die Tatsache verschleiert, dass die größte Quelle der Varianz in unserem System die Lehrpersonen sind (sowohl die Varianz zwischen Lehrpersonen, aber sogar bei ein und derselben Lehrperson kann ihre Wirkung an verschiedenen Tagen und zwischen verschiedenen Fächern deutlich variieren). Worauf es tatsächlich ankommt, ist, dass Lehrpersonen über eine Geisteshaltung verfügen, die sie veranlasst, ihre Wirkung auf das Lernen zu evaluieren. ${ }^{26}$

Fehlt systematische Evaluation, läuft Unterricht beständig Gefahr, die Diskrepanz zwischen Lehrinhalten und erworbenen Fachkompetenzen zu vergrößern. Die folgenden Diagramme veranschaulichen den Sachverhalt:
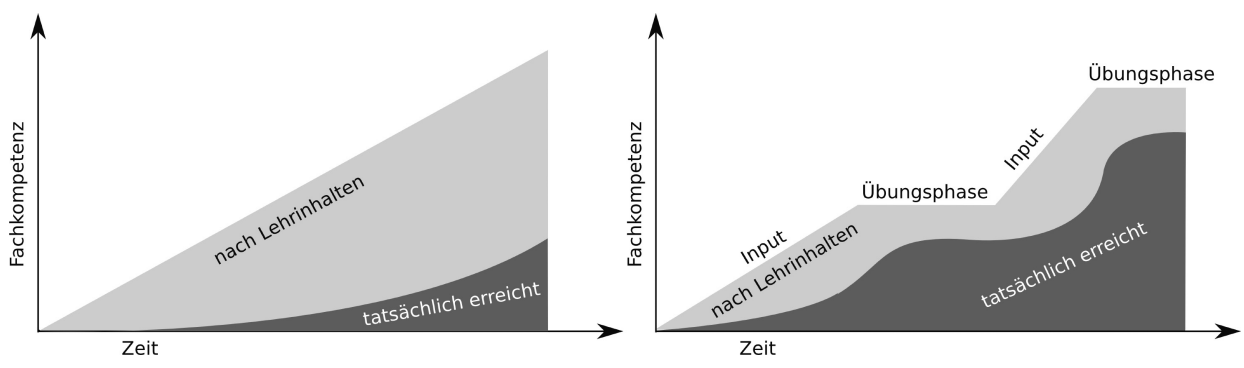

Abbildung 2: Kompetenzerwerb im Unterricht

Erst Evaluation macht sichtbar, ob die Vermittlung von Fachinhalten zum Erwerb von Handlungskompetenz führt oder nicht. Wird durch Evaluation festgestellt, dass Lehrin-

26 Hattie 2014, 17 (Hervorhebungen original). 
halte und erworbene Fachkompetenz auseinanderklaffen, können Übungsphasen und echte Lernzeit die Kluft zwischen fachlichem Input und studentischem Output verringern. Unterricht, der in dieser Form Lernen sichtbar macht, wird seiner eigenen Evaluierung verpflichtet sein und anschließende Unterrichtsgestaltungen an diesen Evaluationsergebnissen orientieren.

Geht man davon aus, dass Lehren auch an Musikhochschulen eine Profession ist ${ }^{27}$ und dass Reflexion und Evaluation auch hier wesentliche Merkmale guten Unterrichts darstellen, rücken zwei Probleme in den Vordergrund. Das erste besteht darin, dass jenseits wohlklingender Studienordnungen und Modulhandbücher ein Großteil des Unterrichts in Musiktheorie an Musikhochschulen Fachinhalten verpflichtet ist und diese auf Unterrichtsstunden verteilt werden. Das zweite besteht darin, dass Stellen in Musiktheorie für das Gelingen von Lehrproben vergeben werden, die in ihrer einseitigen Fixierung auf das Unterrichtsgeschehen problematisch und von vielen Zufällen ${ }^{28}$ abhängig sind. Der zuletzt genannte Umstand wirkt wiederum negativ auf den Unterricht in Methodik des Hauptfachs Musiktheorie/Gehörbildung zurück, weil man gezwungen ist, zwischen gutem Unterricht und gutem Lehrprobenunterricht zu unterscheiden. Denn Unterricht, in dem man Studierenden über einen längeren Zeitraum hilft, Fachkompetenzen zu erwerben, hat wenig gemein mit Unterricht, in dem man versucht, in kurzer Zeit eine Kommission zu überzeugen.

Die erste Lehrprobe (Traviata) wurde seinerzeit von mir an der Hochschule für Musik und Theater München gehalten, ihr verdanke ich meine derzeitige Stelle (C3-Professur im Fach Musiktheorie). Die zweite Lehrprobe (Harmonielehrekarten) habe ich 2000 anlässlich einer Vorstellung für eine C3-Professur an der Hochschule für Musik in Hamburg konzipiert und realisiert, die dritte (Choralsatz) 2014 im Rahmen einer Vorstellung für eine W3-Professur an der Hochschule für Musik in Freiburg. In Hamburg führte die Lehrprobe zu einem Listenplatz, die Freiburger Stelle wurde nicht besetzt, weil sich die Kommission auf keinen Kandidaten einigen konnte. Während ich mit der beschriebenen Choralsatz-Vorgehensweise in München im Rahmen eines Proseminars zur `Regola dell'ottavas seit Jahren sehr gute Erfahrungen mache, war sie in Freiburg ein Thema zur falschen Zeit am falschen Ort.

27 Davon kann man nur ausgehen, wenn man Luhmann in folgendem Punkt widerspricht: »Die pädagogische und didaktische Komponente ist für die Elementarschulen wichtig, aber ihre Bedeutung nimmt ab, sobald Fachunterricht hinzutritt. Man braucht nicht Pädagogik studiert zu haben, um an höheren Schulen Mathematik, Geographie, Geschichte usw. zu unterrichten. Der Fachunterricht ist auch deshalb nicht professionell, weil er eine Übertragung des Wissens auf den Klienten anstrebt, während für Professionen das Gegenteil zutrifft. Kein Arzt will seinen Patienten zum Mediziner, kein Jurist seinen Klienten zum Juristen machen, auch wenn im Beratungsprozeß eine begrenzte Belehrung eine Rolle spielen mag. Auch der Lehrer arbeitet daher nur insofern professionell, als er ein Wissen und Können benutzt, das er nicht lehren, nicht übertragen will. In den höheren Schulen und erst recht in den Universitäten reduziert sich daher die professionelle Komponente auf ein in der Praxis angeeignetes Geschick.« (Luhmann 2002, 151)

28 Im Rahmen von Probeunterricht bei Berufungsverfahren ist ein großes Problem, dass den Unterrichtenden (und manchmal sogar der Kommission) bis zum Beginn des Unterrichts weder die Gruppenzusammensetzung noch das (oft sehr heterogene) Leistungsniveau der Studierenden bekannt sind. 


\section{Literatur}

Bundesministerium für Bildung und Forschung (BMBF; Hg.) (2007), Zur Entwicklung nationaler Bildungsstandards (= Bildungsforschung 1), Bonn/Berlin: o. V., unveränderter Nachdruck 2009, http://www.bmbf.de/pub/zur_entwicklung_nationaler_bildungs standards.pdf

Hattie, John (2013), Lernen sichtbar machen, überarbeitete dt.-sprachige Ausg., besorgt von Wolfgang Beywl und Klaus Zierer, Baltmannsweiler: Schneider Hohengehren [Hattie, John (2009), Visible learning. A Synthesis of over 800 Meta-Analyses Relating to Achievement, London/New York: Routlege].

— (2014): Lernen sichtbar machen für Lehrpersonen, überarbeitete dt.-sprachige Ausg., besorgt von Wolfgang Beywl und Klaus Zierer, Baltmannsweiler: Schneider Hohengehren [Hattie, John (2012), Visible Learning for Teachers. Maximizing impact on Learning, London/New York: Routledge].

Kaiser, Ulrich (2010): „Sokrates und die Musiktheorie», in: Diergarten, Felix / Ludwig Holtmeier / John Leigh / Edith Metzner (Hg.), Musik und ihre Theorien. Clemens Kühn zum 65. Geburtstag, Dresden: Sandstein, 197-202. http://www.kaiser-ulrich. de/Files/Public/Kaiser_SokratesUndMusiktheorie.pdf

- (o.J.), Choralsatz schreiben. http://www.musikanalyse.net/tutorials/choralsatz Kühn, Clemens (2006): Musiktheorie unterrichten - Musik vermitteln. Erfahrungen - Ideen - Methoden. Ein Handbuch, Kassel u. a.: Bärenreiter.

Bosold, Bernhard / Markus Eisele / Maria Jakobs / Karl Rueß / Angelika Scholz / Eberhard Schwefel (o.J.), Kompetenzen und Lernziele. http://lehrerfortbildung-bw.de/faecher/ religion/gym/fb1/1_theorie/komp/3_wassind/2_ziele/

Luhmann, Niklas (2002): Das Erziehungssystem der Gesellschaft, hg. von Dieter Lenzen, Frankfurt a.M.: Suhrkamp.

— (2004): Schriften zur Pädagogik, hg. von Dieter Lenzen, Frankfurt a. M.: Suhrkamp.

— / Eberhard Schorr (1982): Technologie und Selbstreferenz. Fragen an die Pädagogik, Frankfurt am M.: Suhrkamp.

- Eberhard Schorr (1988): Reflexionsprobleme im Erziehungssystem, Frankfurt a. M.: Suhrkamp.

Meyer, Hilbert (2007), Leitfaden Unterrichtsvorbereitung, komplett überarbeitete Neuauflage, Berlin: Cornelsen Scriptor.

— (2011): Was ist guter Unterricht? 8. Aufl., Berlin: Cornelsen Scriptor.

Reich, Kersten (2005): »Konstruktivistische Didaktik auf dem Weg, die Didaktik neu zu erfinden", in: Voß, Reinhard (Hg): LernLust und EigenSinn. Systemisch-konstruktivistische Lernwelten, Heidelberg: Carl Auer, 179-190. http://www.uni-koeln.de/hf/ konstrukt/reich_works/aufsatze/reich_47.pdf

(2014), Methodenpool, Köln. http://methodenpool.uni-koeln.de/

Scheunpflug, Annette (2004): »Das Technologiedefizit - Nachdenken über Unterricht aus systemtheoretischer Perspektive«, in: Lenzen, Dieter (Hg.), Irritationen des Erziehungssystems. Pädagogische Resonanzen auf Niklas Luhmann, Frankfurt a. M.: Suhrkamp, 65-87. 
Weidner, Verena (2012): »'Dieく Musiktheorie `der Musikpädagogik. Systemtheoretische Beobachtungen«, in: Niessen, Anne / Jens Knigge (Hg.), Musikpädagogisches Handeln (= Musikpädagogische Forschung 33), Essen: Die Blaue Eule, 300-315. http:// www.pedocs.de/volltexte/2014/8576/pdf/AMPF_2012_Band_33.pdf

(Druck i. V. a): Musikpädagogik und Musiktheorie. Systemtheoretische Beobachtungen einer problematischen Beziehung (= Perspektiven musikpädagogischer Forschung 2), Münster: Waxmann.

(Druck i. V. b): „Soziologische Systemtheorie und Musikpädagogik. Annäherungen an ein Theorieverhältnis", in: Niessen, Anne / Jens Knigge (Hg.), Theoretische Rahmung und Theoriebildung in der musikpädagogischen Forschung (= Musikpädagogische Forschung 36), Münster: Waxmann. 